\title{
General equilibrium in markets for lemons
}

\author{
João Correia-da-Silva* \\ Universidade do Porto, CEF.UP and Faculdade de Economia, Rua Dr. Roberto Frias, 4200-464 Porto, Portugal
}

\section{A R T I C L E I N F O}

\section{Article history:}

Received 13 June 2010

Received in revised form

10 October 2011

Accepted 13 April 2012

Available online 25 April 2012

\section{Keywords:}

General equilibrium

Asymmetric information

Adverse selection

Uncertain delivery

Delivery rates

\begin{abstract}
A B S T R A C T
This paper studies exchange economies in which agents have differential information about the goods that the other agents bring to the market. To study such a setting, it is useful to distinguish goods not only by their physical characteristics, but also by the agent that brings them to the market. Equilibrium is shown to exist, with agents receiving the cheapest bundle among those that they cannot distinguish from the truthful delivery. An example is presented as an illustration.
\end{abstract}

(C) 2012 Elsevier B.V. All rights reserved.

\section{Introduction}

Economic agents usually trade goods without having perfect knowledge of their characteristics. This applies to firms hiring workers with unknown productivity, to consumers buying used cars with unknown quality and to financial institutions buying assets with unknown return. Each trader enters the market with specific prior knowledge and observation abilities concerning the characteristics of the goods being traded. This is a particular kind of asymmetric information (adverse selection), famous since the seminal contribution of Akerlof (1970).

General equilibrium models with adverse selection have been developed by Prescott and Townsend (1984a,b), Gale (1992, 1996), Bisin and Gottardi (1999, 2006) and Rustichini and Siconolfi (2008), among others. In these models, agents enter the market having private information about their endowments and preferences in each of the possible states of nature. Here, an alternative formulation is considered. Each agent's private information is described by a partition of the set of commodities, such that

\footnotetext{
放 I am grateful to Andrés Carvajal, Carlos Hervés Beloso, Herakles Polemarchakis, an anonymous referee and an Associate Editor for useful comments and suggestions that allowed me to improve the paper, and to Joana Pinho for assisting me with the computations. I also wish to thank the participants in seminars at the Paris School of Economics, U. Vienna, U. Warwick, U. Reading, U. Exeter and U. Porto, in the 2009 European GE Workshop in honor of Andreu Mas-Colell, in the 2009 SAET Conference and in the 2009 UECE Game Theory Meeting in Lisbon. Financial support from CEF.UP, Fundação para a Ciência e Tecnologia and FEDER (research grant PTDC/EGE-ECO/111811/2009) is acknowledged.

* Tel.: +351 225571100; fax: +351225505050.

E-mail address: joao@fep.up.pt.
}

the agent can only distinguish goods that belong to different sets of the partition. This formulation, in the spirit of Akerlof (1970), was proposed by Minelli and Polemarchakis (2000) and Meier et al. (2011).

The distinctive feature of the work of Meier et al. (2011) is the consideration of what they designate as two-sided private information: agents are allowed to have differential information about the goods that the other agents bring to the market (instead of being equally uninformed). ${ }^{1}$ This contrasts with what is assumed in all the previous literature. For example, in the model of Dubey et al. (2005), in which sellers of an asset may or may not default on their promises of future payments, all the buyers receive the same payoff (because deliveries are pooled). If one recognizes that some buyers have superior abilities to identify the sellers who are more likely to default, then it becomes essential to study how and to what extent they are able to exploit their informational advantage.

The model of Meier et al. (2011) is intended for the pursuit of such an investigation. A drawback of their formulation is that it only allows for the opening of markets for classes of goods that everyone can distinguish. For example, if there is an agent in the economy that does not distinguish red cherries from green cherries, then the other agents cannot trade red and green cherries at different prices. The existence of a single uninformed buyer

\footnotetext{
1 Agents are assumed to have perfect information about their endowments, and only have differential information about the characteristics of the goods that are brought to the market by the other agents. This is a natural assumption, based on the observation that the owner of a good typically has superior information about its characteristics.
} 
implies that the sellers of red cherries must charge the same price as the sellers of green cherries. ${ }^{2}$

Here, an alternative framework is proposed. There are markets for each of the goods, which may, therefore, be traded at different prices. Nevertheless, an agent that buys red cherries, and is not able to distinguish red cherries from green cherries, may end up receiving green cherries instead of red cherries (or any mixture of green and red cherries). ${ }^{3}$ Instrumental to this treatment of trade with adverse selection is the concept of delivery rate, adapted from the work of Dubey et al. (2005). If an agent that buys 10 tons of red cherries receives 2 tons of red cherries and 8 tons of green cherries, the corresponding delivery rates are 0.2 and 0.8 . It is assumed that agents take the delivery rates as given. In equilibrium, the anticipated delivery rates must coincide with the actual delivery rates.

As in the model of Prescott and Townsend (1984a,b) and the subsequent literature, we assume that a profit-maximizing firm is responsible for trade intermediation. Taking prices as given, the firm buys the endowments and resells them to the agents. The firm can find it to be in its interest to deliver bundles that are different from those that the agents ordered. In fact, to maximize profits, the firm delivers to each agent the cheapest possible alternative among those that the agent cannot distinguish from the truthful delivery. The firm is able to do this because the abilities to distinguish goods (information partitions of the agents) are commonly known by the agents and by the intermediary firm (all the primitives of the economy are common information). This captures those situations in which sellers are able to figure out whether they are facing an informed or an uninformed buyer.

To sum up, the workings of the economy are summarized as follows. Goods are labeled not only by their physical characteristics, but also by the agent that brings them to the market. There are prices for each of these generalized goods, taken as given by the agents and by the intermediary firm. There are also personalized rates of delivery for each of these generalized goods, chosen by the intermediary firm and taken as given by the agents. An equilibrium is composed by prices, delivery rates, orders and deliveries, such that agents make orders that maximize the utility of the resulting deliveries (given the correctly anticipated delivery rates), which are feasible and maximize the profit of the intermediary firm (among those deliveries that the agents cannot distinguish from the truthful delivery). ${ }^{4}$

The main result of this paper is the existence of equilibrium (Section 2), under very general conditions (the utility functions must be concave and strictly increasing). A characteristic of equilibrium is that agents receive the cheapest bundle among those that they cannot distinguish from truthful delivery, and that the law of one price is verified: different sellers charge the same price for the same good, but may charge different prices for different goods even if these goods are not distinguished by some of the agents (Section 3). To illustrate the main intuitions offered by the model, an example is solved and explained (Section 4). The welfare properties of the model are studied and it is shown that the equilibrium allocation can be Pareto-improved through a system of taxes and subsidies (Section 5). The paper concludes with some remarks (Section 6).

\footnotetext{
2 The differential information of the buyers is modeled as a "picking ability". All buyers pay the same price, but the informed are able to pick the high quality goods, while the uninformed pick at random.

3 An agent guarantees delivery of one out of a set of possibilities. This is closely related to what was termed as "uncertain delivery" by Correia-da-Silva and HervésBeloso (2008, 2009, forthcoming), in a series of papers that study ex-ante trade of contingent goods, with agents having different abilities to verify the occurrence of the exogenous states of nature.

4 It would be interesting to study the implications of allowing the intermediary firm to condition trading on quantity limits, as in the work of Dubey and Geanakoplos (2002). We leave this investigation for future work.
}

\section{The model}

Consider an exchange economy in which a finite number of agents, $i \in \ell=\{1, \ldots, I\}$, trade a finite number of goods, $l \in$ $\mathcal{L}=\{1, \ldots, L\}$.

To capture the usual context in which the seller has superior information about the quality of the goods that he/she brings to the market, it is useful to consider a generalized notion of a good, incorporating in its description the name of the agent that is endowed with the good. This allows us to study markets in which agents may not have the ability to distinguish good cars from bad cars in general, but are able to observe the quality of their own cars.

We refer to good $l$ that is in the initial endowment of agent $i$ as the generalized good $(l, i) \in \mathcal{L} \times \ell$. The initial endowments of agent $i$, defined in terms of these generalized commodities, $f_{i} \in \mathbb{R}_{+}^{L I}$, relate to the usual definition of endowments, $e_{i} \in \mathbb{R}_{+}^{L}$, as follows:

$f_{i} \in \mathbb{R}_{+}^{L I}, \quad$ with $f_{i}^{(l, i)}=e_{i}^{l}$ and $f_{i}^{(l, j)}=0, \forall l, i, j \neq i$.

Similarly, the utility functions in terms of these generalized commodities, $V_{i}$, can be obtained from the usual utility functions, $U_{i}: \mathbb{R}_{+}^{L} \rightarrow \mathbb{R}$, as follows:

$V_{i}: \mathbb{R}_{+}^{L I} \rightarrow \mathbb{R}$

$V_{i}\left(x_{i}\right)=U\left(z_{i}\right), \quad$ where $z_{i}^{l}=\sum_{j \in \ell} x_{i}^{(l, j)}$.

Agents wish to maximize their utility functions, $V_{i}\left(x_{i}\right)$, which are continuous, concave and strictly increasing. ${ }^{5}$

Assumption 1 (Preferences). The utility functions, $V_{i}$, are continuous, concave and strictly increasing.

Each agent has specific abilities to distinguish the different goods that are traded in the market. These observation abilities are described by a partition of the set of generalized goods, $P_{i}$, such that $\left(l^{\prime}, j^{\prime}\right) \in P_{i}(l, j)$ if and only if agent $i$ cannot distinguish good $\left(l^{\prime}, j^{\prime}\right)$ from good $(l, j){ }^{6}$

The inability to distinguish between two goods, $(l, j)$ and $\left(l^{\prime}, j^{\prime}\right)$, implies that an agent that buys certain quantities of $(l, j)$ and $\left(l^{\prime}, j^{\prime}\right)$, say $y_{i}^{(l, j)}$ and $y_{i}^{\left(l^{\prime}, j^{\prime}\right)}$, may receive different quantities, $x_{i}^{(l, j)}$ and $x_{i}^{\left(l^{\prime}, j^{\prime}\right)}$, such that

$x_{i}^{(l, j)}+x_{i}^{\left(l^{\prime}, j^{\prime}\right)}=y_{i}^{(l, j)}+y_{i}^{\left(l^{\prime}, j^{\prime}\right)}$.

More generally, when buying $y_{i}=\left(y_{i}^{(1,1)}, \ldots, y_{i}^{(1, I)}, y_{i}^{(2,1)}, \ldots\right.$, $\left.y_{i}^{(L, I)}\right)$, agent $i$ will receive $x_{i}=\left(x_{i}^{(1,1)}, \ldots, x_{i}^{(1, I)}, x_{i}^{(2,1)}, \ldots, x_{i}^{(L, I)}\right)$ such that (see Box I).

The delivery matrix, $k_{i}$, is endogenous (equilibrating variable). The delivery matrices that are compatible with the abilities of agent $i$ to distinguish commodities are such that, for each $(l, j)$,

$$
\begin{gathered}
\sum_{\left(l^{\prime}, j^{\prime}\right) \in P_{i}(l, j)} k_{i}^{\left(l^{\prime}, j^{\prime}\right),(l, j)}=1 \text { and } \\
k_{i}^{\left(l^{\prime}, j^{\prime}\right),(l, j)}=0, \forall\left(l^{\prime}, j^{\prime}\right) \notin P_{i}(l, j) .
\end{gathered}
$$

The set of matrices that satisfy these conditions is denoted $K_{i}$, and $K=\prod_{i \in \ell} K_{i}{ }^{7}$

\footnotetext{
5 By strictly increasing, it is meant that an increase in consumption of any of the goods is strictly desired by the agents: $x_{i} \geq x_{i}^{\prime}$ and $x_{i} \neq x_{i}^{\prime}$ implies that $V_{i}\left(x_{i}\right)>V_{i}\left(x_{i}^{\prime}\right)$

6 It is assumed that agents are not able to share information. This intends to model situations in which sellers are not able to credibly announce the quality of the goods that they bring to the market (like in the used car market).

7 The sum of the elements of a column must be equal to 1 , and those elements that correspond to goods that can be distinguished from the good in the diagonal must be equal to 0 .
} 
$\left[\begin{array}{c}x_{i}^{(1,1)} \\ \vdots \\ x_{i}^{(1, I)} \\ x_{i}^{(2,1)} \\ \vdots \\ x_{i}^{(L, I)}\end{array}\right]=\left[\begin{array}{cccccc}k_{i}^{(1,1),(1,1)} & \cdots & k_{i}^{(1,1),(1, I)} & k_{i}^{(1,1),(2,1)} & \cdots & k_{i}^{(1,1),(L, I)} \\ \vdots & \ddots & \vdots & \vdots & \ddots & \vdots \\ k_{i}^{(1, I),(1,1)} & \cdots & k_{i}^{(1, I),(1, I)} & k_{i}^{(1, I),(2,1)} & \cdots & k_{i}^{(1, I),(L, I)} \\ k_{i}^{(2,1),(1,1)} & \cdots & k_{i}^{(2,1),(1, I)} & k_{i}^{(2,1),(2,1)} & \cdots & k_{i}^{(2,1),(L, I)} \\ \vdots & \ddots & \vdots & \vdots & \ddots & \vdots \\ k_{i}^{(L, I),(1,1)} & \cdots & k_{i}^{(L, I),(1, I)} & k_{i}^{(L, I),(2,1)} & \cdots & k_{i}^{(L, I),(L, I)}\end{array}\right] \cdot\left[\begin{array}{c}y_{i}^{(1,1)} \\ \vdots \\ y_{i}^{(1, I)} \\ y_{i}^{(2,1)} \\ \vdots \\ y_{i}^{(L, I)}\end{array}\right]$,
where $k_{i}^{\left(l^{\prime}, j^{\prime}\right),(l, j)} \geq 0$ denotes the number of units of good $\left(l^{\prime}, j^{\prime}\right)$ that agent $i$ receives for each unit of good $(l, j)$ that he/she buys. Box I.

It should be clear that if agent $i$ is able to distinguish all the commodities, that is, if $P_{i}(l, j)=\{(l, j)\}, \forall(l, j)$, then $K_{i}$ has a single element (the identity matrix). A perfectly informed agent always receives exactly the bundle that he/she orders.

For simplicity, it is assumed that agents have perfect information about their own endowments. ${ }^{8}$

\section{Assumption 2 (Perfect Information About Own Endowments).}

$\forall(i, l): P_{i}(l, i)=\{(l, i)\}$.

Only goods that exist are priced and traded in the market. Such goods are those for which $\sum_{i \in \ell} f_{i}^{(l, j)}>0$, a condition which is equivalent to $f_{j}^{(l, j)}>0$. Absence of some goods implies straightforward modifications of the spaces in which $f_{i}, k_{i}, V_{i}$ and $P_{i}$ are defined.

The set of goods that exist is denoted by $\mathcal{M} \subseteq \mathcal{L} \times \ell$, and the number of goods that exist by $M \leq L I$. When the classical interiority assumption holds $\left(e_{i} \gg 0, \forall i \in \ell\right)$, all the goods are traded in the market and, therefore, $\mathcal{M}=\mathcal{L} \times \ell$ and $M=L I$.

Taking prices, $p \in \Delta^{M}$, and delivery rates, $k_{i} \in K_{i}$, as given, agent $i$ trades his/her initial endowments, $f_{i} \in \mathbb{R}_{+}^{M}$, for a bundle, $y_{i} \in \mathbb{R}_{+}^{M}$, that maximizes utility, $V_{i}\left(k_{i} y_{i}\right)$, among those that satisfy the budget restriction, $y_{i} \in B_{i}(p)$, with

$B_{i}(p)=\left\{y_{i} \in \mathbb{R}_{+}^{M}: p \cdot y_{i} \leq p \cdot f_{i}\right\}$.

Notice that Assumption 2 guarantees individual rationality of participating in the market. An agent can always buy back his/her own endowments.

A profit-maximizing firm is responsible for trade intermediation. ${ }^{9}$ Without loss of generality, it can be assumed that the firm buys the total endowments in the economy, as the firm can resell the goods at the same price. When acting as a seller, the firm can deliver a bundle that is different from the bundle that an agent orders. To maximize profits, the firm delivers the cheapest possible alternative among those that the agent cannot distinguish from truthful delivery.

It may seem that the intermediary firm is being given information that the agents in the economy do not have themselves. In fact, the intermediary firm only uses information that all the agents have. We have assumed that the economy is common information, therefore, all the agents and the intermediary firm know the endowments and information partitions of everyone. Therefore, having the privilege of mediating all the trade, the intermediary firm knows exactly what it is buying from each agent. And as agents cannot cheat the intermediary firm about the goods that they are

\footnotetext{
8 In a dynamic setup, agents would probably have incentives to communicate truthfully the characteristics of the goods they are selling in order to build or preserve their reputation. Since communication is not allowed, this model is more satisfactory for modeling situations in which there are no repeated interactions.

9 Profit-maximization is assumed, although this behavior may harm some individuals who can be stockholders.
}

delivering, there are no incentives for them to misreport to the intermediary firm the characteristics of each good. ${ }^{10}$ When choosing what bundle to deliver to each agent, the intermediary firm also makes use of information that is available to everyone: the information partitions of each agent.

Definition 1 (Equilibrium). An equilibrium of the economy $\mathcal{E} \equiv$ $\left\{f_{i}, V_{i}, P_{i}\right\}_{i \in \ell}$ is composed of a price system, $p^{*} \in \Delta_{+}^{M}$, individual choices, $y^{*}=\left(y_{1}^{*}, \ldots, y_{I}^{*}\right) \in \mathbb{R}_{+}^{I M}$, personalized delivery rates, $k^{*}=$ $\left(k_{1}^{*}, \ldots, k_{I}^{*}\right) \in K$, and the resulting allocation, $x^{*}=\left(x_{1}^{*}, \ldots, x_{I}^{*}\right) \in$ $\mathbb{R}_{+}^{I M}$, which satisfy:

$$
\begin{aligned}
& y_{i}^{*} \in \operatorname{argmax}_{y_{i} \in B_{i}\left(p^{*}\right)} V_{i}\left(k_{i}^{*} y_{i}\right), \forall i \text { [utility maximization]; } \\
& k^{*} \in \operatorname{argmin}_{k \in K}\left\{p^{*} \cdot \sum_{i \in \ell} k_{i} \overline{1}\right\} \text { [profit maximization] }{ }^{11} ; \\
& x_{i}^{*}=k_{i}^{*} y_{i}^{*}, \forall i \text { [resulting deliveries]; } \\
& \sum_{i \in \ell} x_{i}^{*} \leq \sum_{i \in \ell} f_{i} \text { [feasibility]. }
\end{aligned}
$$

Theorem 1 (Existence of Equilibrium). Under Assumptions 1 and 2, there exists an equilibrium of the economy.

Proof. Consider, for now, bounded choice sets. For choices in the upper bound to imply aggregate excess delivery, let $E=$ $\sum_{(l, j) \in \mathcal{M}} f_{j}^{(l, j)}+1$ and define the following convex and bounded choice sets:

$\bar{Y}_{i}=\left\{y_{i} \in \mathbb{R}_{+}^{M}: y_{i}^{(l, j)} \leq E, \forall(l, j)\right\}$.

The budget set of agent $i$, in this bounded economy, is

$\bar{B}_{i}(p)=\left\{y_{i} \in \bar{Y}_{i}: p \cdot y_{i} \leq p \cdot f_{i}\right\}$.

Let $\psi_{i}(y, p, k)=\operatorname{argmax}_{z_{i} \in \bar{B}_{i}(p)}\left\{V_{i}\left(k_{i} z_{i}\right)\right\}$.

The utility function, $V_{i}\left(k_{i} y_{i}\right)$, is continuous with respect to both $k_{i}$ and $y_{i}$.

As long as $p \cdot f_{i}>0$ (which is always the case for $p \gg 0$ ), the budget correspondence, $\bar{B}_{i}(p)$, is continuous with non-empty compact values.

When this is the case, we know, from Berge's Maximum Theorem, ${ }^{12}$ that the demand correspondence, $\psi_{i}(y, k, p)$, is upper hemicontinuous with nonempty compact values. It is also convexvalued, because $V_{i}$ is concave and $k_{i}$ is constant.

10 If an agent is endowed with $e^{r}$ red cherries and $e^{g}$ green cherries, the firm may not be able to distinguish the red from the green cherries, but knows, however, that the agent is delivering $e^{r}$ red cherries and $e^{g}$ green cherries. The agent could, nevertheless, cheat the firm by labeling some of the red cherries as green and the same number of the green ones as red. It seems that agents have no incentives to do this, but a rigorous investigation of this possibility would require a more sophisticated model. This is left for future work.

11 Since the profit-maximizing delivery matrix does not depend on the orders, we consider a diagonal vector, $\overline{1}$, instead of the equilibrium order, $y_{i}^{*}$, in the argument for the minimization. Otherwise, the null coordinates of $y_{i}^{*}$ would lead to a multiplicity of solutions, which could be interpreted, economically, as noncredible threats.

12 See, for example, Aliprantis and Border (2006). 
Let $\Delta_{\epsilon}^{M}=\left\{p \in \Delta^{M}: p \geq \epsilon\right\}$. We will start by finding a fixed point with strictly positive prices, on $\Delta_{\epsilon}^{M}$, and then let $\epsilon \rightarrow 0$ to obtain a sequence of fixed points.

Let $\psi_{p}^{\epsilon}(y, p, k)=\operatorname{argmax}_{q \in \Delta_{\epsilon}^{M}}\left\{q \cdot \sum_{i \in \ell}\left(k_{i} y_{i}-f_{i}\right)\right\}$.

And let $\psi_{k_{i}}(y, p, k)=\operatorname{argmin}_{d_{i} \in K_{i}}\left\{p \cdot d_{i} \overline{1}\right\}$.

All these correspondences $\left(\psi_{i}, \psi_{p}^{\epsilon}\right.$ and $\left.\psi_{k_{i}}\right)$ are upper hemicontinuous with nonempty compact and convex values. Therefore, the product correspondence, $\psi^{\epsilon}=\prod_{i=1}^{I} \psi_{i} \times \psi_{p}^{\epsilon} \times \prod_{i=1}^{I} \psi_{k_{i}}$, also is.

Applying the Theorem of Kakutani, we find that there exists a fixed point of $\psi^{\epsilon}$, that we denote by $\left(y^{\epsilon}, p^{\epsilon}, k^{\epsilon}\right)$. Considering a sequence, $\left\{\epsilon^{n}\right\}_{n \in \mathbb{N}}$, that converges to zero, we obtain a sequence of fixed points, $\left\{\left(y^{n}, p^{n}, k^{n}\right)\right\}_{n \in \mathbb{N}}$. The sequence is contained in a compact set, therefore a subsequence converges to $\left(y^{*}, p^{*}, k^{*}\right)$.

We want to consider this subsequence and verify that its limit is an equilibrium.

Suppose that the sequence of prices in the interior of the simplex, $\left\{p^{n}\right\}$, converges to a price on the border of the simplex. There is at least one agent whose income does not tend to zero $\left(p^{*} \cdot f_{i}>0\right)$, and therefore whose demand (which is u.h.c.) is driven to the bound of the choice set (recall that utility is strictly increasing). This implies that, for sufficiently large $n$ and in the limit, there is aggregate excess delivery of at least some good:

$\exists(l, j): \sum_{i \in \ell} x_{i}^{(l, j) *}=\sum_{i \in \ell} \sum_{\left(l^{\prime}, j^{\prime}\right)} k_{i}^{(l, j),\left(l^{\prime}, j^{\prime}\right) *} y_{i}^{\left(l^{\prime}, j^{\prime}\right) *}>\sum_{i \in \ell} f_{i}^{(l, j)}$.

The budget restrictions imply that $p^{n} \cdot \sum_{i \in \ell} y_{i}^{n} \leq p^{n} \cdot \sum_{i \in \ell} f_{i}$, and the definition of $\psi_{k_{i}}$ implies that $p^{n} \cdot \sum_{i \in \ell} k_{i}^{n} y_{i}^{n} \leq p^{n} \cdot \sum_{i \in \ell} y_{i}^{n}$ (the identity matrix is enough for equality). In the limit

$p^{*} \cdot \sum_{i \in \ell} x_{i}^{*}=p^{*} \cdot \sum_{i \in \ell} k_{i}^{*} y_{i}^{*} \leq p^{*} \cdot \sum_{i \in \ell} y_{i}^{*}=p^{*} \cdot \sum_{i \in \ell} f_{i}$.

Start by supposing that, in the limit, there is a single good, $(l, j)$, with maximal excess delivery. From the definition of $\psi_{p}$, the price of this good tends to 1 , and the remaining goods have vanishing prices. Therefore, condition (1) implies that there is no excess delivery (contradiction):

$p^{*} \cdot \sum_{i \in \ell} x_{i}^{*} \leq p^{*} \cdot \sum_{i \in \ell} f_{i} \Rightarrow \sum_{i \in \ell} x_{i}^{*(l, j)} \leq \sum_{i \in \ell} f_{i}^{(l, j)}$.

Now consider the general case. Suppose that, in the limit, there is a set of goods, $G$, tied for the maximal excess delivery. In the limit, the prices of these goods must add to 1 (from the definition of $\psi_{p}$ ), while the prices of the remaining goods become null. Condition (1) implies that

$$
\begin{aligned}
p^{*} \cdot \sum_{i \in \ell} x_{i}^{*} & \leq p^{*} \cdot \sum_{i \in \ell} f_{i} \Rightarrow \sum_{(l, j) \in G} \sum_{i \in \ell} p^{(I, j) *} x_{i}^{*(l, j)} \\
& \leq \sum_{(I, j) \in G} \sum_{i \in \ell} p^{(l, j) *} f_{i}^{(l, j)},
\end{aligned}
$$

which contradicts the hypothesis of excess delivery of all the goods $(l, j) \in G$.

Therefore $p^{*} \gg 0$ and there is not excess delivery of any good ( $x^{*}$ is feasible). Existence of equilibrium in the bounded economy is established.

To check that this is an equilibrium when the bounds on the choice sets are removed, we must verify that the individual choices remain unaltered.

Observe that the bound on the choice sets is large enough for the individual choices, $y_{i}^{*}$, to be in the interior of $\bar{Y}_{i}$ (otherwise we would not have feasibility). Since preferences are convex, we are sure that the bounds are not binding. If there were a strictly better choice outside $\bar{Y}_{i}$, then there would also be a strictly better choice in the frontier of $\bar{Y}_{i}$.

We have established that, under general conditions, equilibrium exists.

\section{Characteristics of equilibrium}

A first property of the equilibrium allocation results directly from the definition of equilibrium. Each agent receives one of the cheapest bundles among those that he/she cannot distinguish from the truthful delivery. ${ }^{13}$

\section{Proposition 1.}

$p^{*} \cdot x_{i}^{*}=\min _{k_{i} \in K_{i}}\left\{p^{*} \cdot k_{i} y_{i}^{*}\right\}$.

In equilibrium, prices are strictly positive and there is no free disposal.

\section{Proposition 2.}

$p^{*} \gg 0$ and $\sum_{i \in \ell} x_{i}^{*}=\sum_{i \in \ell} f_{i}$.

Proof. In the proof of Theorem 1, it was established that $p^{*} \gg 0$.

If, for some $(l, j)$, we had $\sum_{i \in \ell} x_{i}^{(l, j) *}<\sum_{i \in \ell} f_{i}^{(l, j)}$, then, from the definition of $\psi_{p}^{\epsilon}$, we would have $p^{(l, j) *}=0$. Contradiction.

Each agent receives a bundle that costs the same as the bundle that he/she bought.

\section{Proposition 3.}

$\forall i \in \ell: p^{*} \cdot x_{i}^{*}=p^{*} \cdot y_{i}^{*}$.

Proof. From Proposition 1, we know that an agent cannot receive something more expensive than what he/she bought. Then, from exact feasibility (Proposition 2) it follows that all agents receive a bundle that costs the same as the bundle that they bought. If some agent received something cheaper, another agent would have to be receiving something more expensive, which would be a contradiction.

An agent who does not distinguish between two goods prefers to buy the cheapest. Therefore, two goods that the agents do not distinguish (except for the owners, who surely distinguish their endowments) and that are traded in the market (some agent other than the owner buys a positive quantity) are traded at the same price.

Proposition 4. If $\left(l^{\prime}, j^{\prime}\right) \in P_{i}(l, j)$ and $y_{i}^{(l, j) *}>0$, then $p^{(l, j) *} \leq$ $p^{\left(l^{\prime}, j^{\prime}\right) *} \cdot{ }^{14}$

Proof. Suppose that $p^{(l, j) *}>p^{\left(l^{\prime}, j^{\prime}\right) *}$, and that $y_{i}^{(l, j) *}>0$. Replacing good $(l, j)$ by good $\left(l^{\prime}, j^{\prime}\right)$ in the bundle that is bought by agent $i, y_{i}^{*}$, we construct a bundle that is cheaper and that is undistinguishable from $y_{i}^{*}$. In equilibrium, agent $i$ must be receiving, therefore, a bundle that is cheaper than $y_{i}^{*}$. This contradicts Proposition 3 .

In the case in which buyers do not distinguish the original owners of the goods, the law of one price is verified (if the same commodity is sold by two agents, then it is sold at the same price).

Proposition 5. If $\exists i, i^{\prime}, j, j^{\prime}$ and $l$ such that $\left(l, j^{\prime}\right) \in P_{i}(l, j) \cap P_{i^{\prime}}(l, j)$, $y_{i}^{(l, j) *}>0$ and $y_{i^{\prime}}^{\left(l, j^{\prime}\right) *}>0$, then $p^{\left(l, j^{\prime}\right) *}=p^{(l, j) *}$.

Notice that this does not mean that, as in the model of Meier et al. (2011), different goods that some agents cannot distinguish are traded at the same price. What is stated is that the same good is traded by different agents at the same price.

\footnotetext{
13 It should be clear that this adverse delivery is anticipated by the agents.

14 If we also have $y_{i}^{\left(l^{\prime}, j^{\prime}\right) *}>0$, then $p^{(l, j) *}=p^{\left(l^{\prime}, j^{\prime}\right) *}$.
} 


\section{An example}

In this section, an example is presented as an illustration. ${ }^{15}$ Here the notation is simpler than in the previous section, because it will not be necessary to deal with generalized goods in a formal way.

In the first scenario that is analyzed, an agent that does not distinguish two goods is not able to consume the high quality good, in spite of being willing to pay any price for a small quantity of this good. In the second case, the two goods are traded at the same equilibrium price. The uninformed agent receives both goods, with delivery rates determined by the leftovers of the informed agents.

\subsection{Cherry picking}

Three individuals, $\ell=\{1,2,3\}$, trade three commodities, $\mathcal{L}=$ $\{0, r, g\}$, that we can think of as 'money', 'red cherries' and 'green cherries'.

Agent 1 is endowed with 'money', agent 2 with 'red cherries' and agent 3 with 'green cherries':

$\left\{\begin{array}{l}e_{1}=(12,0,0) \\ e_{2}=(0,12,0) \\ e_{3}=(0,0,12) .\end{array}\right.$

All agents prefer 'green cherries' to 'red cherries'. Agent 2 does not like 'red cherries' at all. Preferences are described by the following utility functions ${ }^{16}$ :

$$
\left\{\begin{array}{l}
U_{1}\left(x_{1}\right)=\ln \left(x_{1}^{0}\right)+\ln \left(x_{1}^{r}\right)+2 \ln \left(x_{1}^{g}\right) \\
U_{2}\left(x_{2}\right)=\ln \left(x_{2}^{0}\right)+2 \ln \left(x_{2}^{g}\right) \\
U_{3}\left(x_{3}\right)=\ln \left(x_{3}^{0}\right)+\ln \left(x_{3}^{r}\right)+2 \ln \left(x_{3}^{g}\right) .
\end{array}\right.
$$

There is asymmetric information because agent 1 cannot distinguish 'red cherries' from 'green cherries' while agents 2 and 3 are able to distinguish the three commodities:

$$
\left\{\begin{array}{l}
P_{1}=\{\{0\},\{r, g\}\} \\
P_{2}=\{\{0\},\{r\},\{g\}\} \\
P_{3}=\{\{0\},\{r\},\{g\}\} .
\end{array}\right.
$$

Agents 2 and 3 are perfectly informed, and therefore receive exactly what they buy. On the other hand, agent 1 can buy either 'red cherries' or 'green cherries', but must accept whatever cherries are delivered (because agent 1 cannot distinguish the two goods). ${ }^{17}$

Since agent 2 will not buy 'red cherries', his/her budget restriction is (the price of 'money' is normalized to $p^{0}=1$ ):

$p^{0} x_{2}^{0}+p^{r} x_{2}^{r}+p^{g} x_{2}^{g}=p^{r} e_{2}^{r} \Rightarrow x_{2}^{0}+p^{g} x_{2}^{g}=12 p^{r}$.

The optimality condition implies equality of the ratios between marginal utility and price, for each good demanded:

$x_{2}^{0}=\frac{1}{2} p^{g} x_{2}^{g}$.

\footnotetext{
15 The example is adapted from Meier et al. (2011). The preferences, endowments and information are maintained to highlight the fact that the equilibrium concept used here leads to qualitatively different solutions.

16 Precisely, we consider that agent 1 has lexicographic preferences. First, the agent maximizes $U_{1}\left(x_{1}\right)=\ln \left(x_{1}^{0}\right)+\ln \left(x_{1}^{r}\right)+2 \ln \left(x_{1}^{g}\right)$. Then, in the case of a tie (which occurs among all bundles with $x_{1}^{g}=0$ ), agent 1 is assumed to maximize $u_{1}\left(x_{1}\right)=\ln \left(x_{1}^{0}\right)+\ln \left(x_{1}^{r}\right)$. The fact that utility is equal to $-\infty$ in the boundary of the consumption set is cumbersome, but notice that the same preferences can be represented by Cobb-Douglas utility functions such as $U_{i}\left(x_{i}\right)=$ $\left(x_{i}^{0}\right)^{0.25}\left(x_{i}^{r}\right)^{0.25}\left(x_{i}^{g}\right)^{0.5}$. In any case, the utility functions are not strictly increasing as assumed in Section 2 to establish existence of equilibrium. In Appendix A.1, the utility functions are modified to $U_{i}\left(x_{i}\right)=\ln \left(x_{i}^{0}+\epsilon\right)+\ln \left(x_{i}^{r}+\epsilon\right)+2 \ln \left(x_{i}^{g}+\epsilon\right)$ and an asymptotic analysis is conducted for $\epsilon \rightarrow 0$ to show that the results are robust to a perturbation that yields strictly increasing utility functions.

17 Observe that there is an infinite number of possibilities for the delivery of 10 'cherries' (10 'red' and 0 'green', 5 'red' and 5 'green', 7 'red' and 3 'green', etc.).
}

From the budget restriction and the optimality condition, we find the demand of agent 2 :

$\left(x_{2}^{0}, x_{2}^{r}, x_{2}^{g}\right)=\left(4 p^{r}, 0,8 \frac{p^{r}}{p^{g}}\right)$.

Similarly, we can obtain the demand function of agent 3:

$$
\begin{aligned}
& \left\{\begin{array}{l}
x_{3}^{0}+p^{r} x_{3}^{r}+p^{g} x_{3}^{g}=12 p^{g} \\
x_{3}^{0}=p^{r} x_{3}^{r}=0.5 p^{g} x_{3}^{g}
\end{array} \Rightarrow\left(x_{3}^{0}, x_{3}^{r}, x_{3}^{g}\right)\right. \\
& =\left(3 p^{g}, 3 \frac{p^{g}}{p^{r}}, 6\right) .
\end{aligned}
$$

Looking at the demand of agents 2 and 3 for 'green cherries', we find that $p^{r}<p^{g}$, otherwise there would be excess demand. More precisely:

$8 \frac{p^{r}}{p^{g}}+6 \leq 12 \Rightarrow p^{r} \leq 0.75 p^{g}$.

Suppose that agent 1 buys a quantity $x_{1}^{r g}$ of 'cherries' (guarantees delivery of 'red cherries' and 'green cherries' such that $x_{1}^{r}+x_{1}^{g}=$ $\left.x_{1}^{r g}\right)$. If 'red cherries' are cheaper than 'green cherries', then the agent should receive only 'red cherries', and no 'green cherries'.

Following this reasoning, since $p^{r}<p^{g}$, then $x_{1}^{g}=0$ and $x_{1}^{r}=$ $x_{1}^{r g}$. Assuming that agent 1 is aware of this (delivery rates are anticipated and taken as given), we can find his/her demand function ${ }^{18}$ :

$\left\{\begin{array}{l}x_{1}^{0}+p^{r} x_{1}^{r}=12 \\ x_{1}^{0}=p^{r} x_{1}^{r}\end{array} \Rightarrow\left\{\begin{array}{l}x_{1}^{0}+x_{1}^{0}=12 \Rightarrow x_{1}^{0}=6 \\ p^{r} x_{1}^{r}+p^{r} x_{1}^{r}=12 \Rightarrow x_{1}^{r}=\frac{6}{p^{r}} .\end{array}\right.\right.$

For demand to equal supply:

$\left\{\begin{array}{l}x_{1}^{0}+x_{2}^{0}+x_{3}^{0}=12 \Rightarrow 4 p^{r}+3 p^{g}=6 \\ x_{1}^{r}+x_{2}^{r}+x_{3}^{r}=12 \Rightarrow \frac{6}{p^{r}}+3 \frac{p^{g}}{p^{r}}=12 \\ x_{1}^{g}+x_{2}^{g}+x_{3}^{g}=12 \Rightarrow 8 \frac{p^{r}}{p^{g}}=6 \Rightarrow p^{r}=0.75 p^{g} .\end{array}\right.$

These equations allow the determination of equilibrium prices:

$p^{*}=\left(p^{0}, p^{r}, p^{g}\right)=(1,0.75,1)$.

The allocation is, therefore $\left(y^{*}=x^{*} \text { yields truthful deliveries }\right)^{19}$,

$\left\{\begin{array}{l}x_{1}^{*}=\left(6, \frac{6}{p^{r}}, 0\right)=(6,8,0) \\ x_{2}^{*}=\left(4 p^{r}, 0,8 \frac{p^{r}}{p^{g}}\right)=(3,0,6) \\ x_{3}^{*}=\left(3 p^{g}, 3 \frac{p^{g}}{p^{r}}, 6\right)=(3,4,6) .\end{array}\right.$

The major differences with respect to the solution obtained by Meier et al. (2011) are that, here, the different types of 'cherries' are traded at different prices and the uninformed agent is not able to consume any 'green cherries'.

\footnotetext{
18 Agent 1 prefers any interior bundle $\left(x_{1} \gg 0\right)$ to a bundle that is in the frontier of the consumption set. But he/she cannot get any 'green cherries' and, therefore, $U_{1}$ becomes infinitely negative. Recall that among bundles with $x_{1}^{g}=0$ (in the boundary of the consumption set), the preferences of agent 1 are described by $u_{1}\left(x_{1}\right)=\ln \left(x_{1}^{0}\right)+\ln \left(x_{1}^{r}\right)$

19 It is assumed that agents are not able to form coalitions and share information. This captures the situations in which the sellers are not able to credibly announce the quality of the goods that they bring to the market.
} 


\subsection{Modified cherry picking}

Is it always the case that agent 1 does not consume 'green cherries'? In this modified example, we find that agent 1 can consume 'green cherries' if the incentives for agents 2 and 3 to deliver only 'red cherries' disappear.

This occurs if 'green cherries' become much more abundant than 'red cherries'. Let us double the endowments of agent 3 ('green cherries'):

$\left\{\begin{array}{l}e_{1}=\{12,0,0\} \\ e_{2}=\{0,12,0\} \\ e_{3}=\{0,0,24\} .\end{array}\right.$

The demand of agent 2 remains unaltered:

$\left\{\begin{array}{l}x_{2}^{0}+p^{g} x_{2}^{g}=12 p^{r} \\ x_{2}^{0}=0.5 p^{g} x_{2}^{g}\end{array} \Rightarrow\left(x_{2}^{0}, x_{2}^{r}, x_{2}^{g}\right)=\left(4 p^{r}, 0,8 \frac{p^{r}}{p^{g}}\right)\right.$,

while the demand of agent 3 doubles:

$$
\begin{aligned}
& \left\{\begin{array}{l}
x_{3}^{0}+p^{r} x_{3}^{r}+p^{g} x_{3}^{g}=24 p^{g} \\
x_{3}^{0}=x_{3}^{r} p^{r}=0.5 x_{3}^{g} p^{g}
\end{array} \Rightarrow\left(x_{3}^{0}, x_{3}^{r}, x_{3}^{g}\right)\right. \\
& =\left(6 p^{g}, 6 \frac{p^{g}}{p^{r}}, 12\right) .
\end{aligned}
$$

Now the demand of agents 2 and 3 for 'green cherries' does not exceed supply as long as $p^{r} \leq 1.5 p^{g}$ :

$x_{2}^{g}+x_{3}^{g}=8 \frac{p^{r}}{p^{g}}+12 \leq 24 \Rightarrow p^{r} \leq 1.5 p^{g}$.

There are three possibilities: (a) the price of 'green cherries' is higher than the price of 'red cherries' and thus agent 1 only consumes 'red cherries' (as in the previous example); (b) the price of 'red cherries' is higher than the price of 'green cherries' and thus agent 1 only consumes 'green cherries'; (c) the prices of 'green cherries' and 'red cherries' coincide.

In case (a), there would be excess supply of 'green cherries', as aggregate consumption is lower than 20 :

$x_{1}^{g}+x_{2}^{g}+x_{3}^{g}=0+8 \frac{p^{r}}{p^{g}}+12<20$.

In case (b), there would be excess supply of 'red cherries', as aggregate consumption is lower than 6 :

$x_{1}^{r}+x_{2}^{r}+x_{3}^{r}=0+0+6 \frac{p^{g}}{p^{r}}<6$.

Thus, in equilibrium we must have case (c): $p^{r}=p^{g}=p^{r g}$. Thus:

$\left\{\begin{array}{l}x_{2}=\left(4 p^{r g}, 0,8\right) \\ x_{3}=\left(6 p^{r g}, 6,12\right)\end{array} \Rightarrow x_{2}+x_{3}=\left(10 p^{r g}, 6,20\right)\right.$.

The only candidate for an equilibrium allocation gives agent 1 the following consumption bundle: $x_{1}=\left(12-10 p^{r g}, 6,4\right)$.

To check whether this is an equilibrium, we need to find the demand of agent 1 . A problem that we face is that agent 1 , through his/her demand, may influence the quality of the 'cherries' (the proportion between 'red' and 'green cherries').

It is assumed that he/she takes the delivery rates of 'red' and 'green cherries' as given. In equilibrium, this proportion must be fulfilled (otherwise it would not be an equilibrium). Since we already have a single candidate for the equilibrium consumption of agent $1, x_{1}=\left(12-10 p^{r g}, 6,4\right)$, we must assume that agent 1 expects to receive $60 \%$ 'red cherries' and $40 \%$ 'green cherries'.

The utility and the maximization condition of agent 1 are (with $\left.x_{1}^{r g}=x_{1}^{r}+x_{1}^{g}\right)$

$$
\begin{aligned}
u_{1}\left(x_{1}\right)= & \ln \left(x_{1}^{0}\right)+\ln \left(0.6 x_{1}^{r g}\right) \\
& +2 \ln \left(0.4 x_{1}^{r g}\right) \Rightarrow x_{1}^{0}=\frac{1}{3} p^{r g} x_{1}^{r g} .
\end{aligned}
$$

Putting this together with the budget restriction, the demand is obtained:

$\left\{\begin{array}{l}x_{1}^{0}+p^{r g} x_{1}^{r g}=12 \\ x_{1}^{0}=\frac{1}{3} p^{r g} x_{1}^{r g}\end{array} \Rightarrow\left(x_{1}^{0}, x_{1}^{r}, x_{1}^{g}\right)=\left(3,0.6 \frac{9}{p^{r g}}, 0.4 \frac{9}{p^{r g}}\right)\right.$.

For the demand of 'money' to equal the supply

$x_{1}^{0}+x_{2}^{0}+x_{3}^{0}=12 \Rightarrow 3+4 p^{r g}+6 p^{r g}=12 \Rightarrow p^{r g}=0.9$.

The equilibrium prices are, therefore, $p^{*}=(1,0.9,0.9)$, and the allocation is

$\left\{\begin{array}{l}x_{1}^{*}=(3,6,4) \\ x_{2}^{*}=(3.6,0,8) \\ x_{3}^{*}=(5.4,6,12) .\end{array}\right.$

In this case, agent 1 consumes both 'red cherries' and 'green cherries', which are traded at the same price. Agents 2 and 3 optimize by delivering 3.6 units of 'red cherries' and 5.4 units of 'green cherries' to agent 1 .

The quantities of 'red' and 'green cherries' that agent 1 orders $\left(y_{1}^{r}\right.$ and $y_{1}^{g}$ ) are irrelevant, given that they add to 10 . If $y^{*}=x^{*}$, there is truthful delivery. In any case, the delivery rates adjust to be such that delivery is surely that calculated above: $x_{1}^{r}=6$ and $x_{1}^{g}=4$.

Observe that the increase in the endowment of 'green cherries' from 12 to 24 units led to a qualitatively different solution. Increasing from 12 to 13 diminishes the price of the 'green cherries' and increases the price of the 'red cherries', but the price of the 'green cherries' remains higher and, therefore, the uninformed agent is still only able to consume 'red cherries'. By straightforward calculations, it can be found that as the endowment of 'green cherries' hits 16 , the prices of the two kinds of cherry become equal, but the uninformed agent still receives only 'red cherries'. The solution, in this case, is

$$
\left\{\begin{array}{l}
p^{*}=(1,0.75,0.75) \\
x_{1}^{*}=(6,8,0) \\
x_{2}^{*}=(3,0,8) \\
x_{3}^{*}=(3,4,8) .
\end{array}\right.
$$

Then, as the endowment of 'green cherries' increases further 'red cherries' become more valuable than 'green cherries' because the scarcity effect overweights the quality effect. The uninformed agent becomes able to consume some 'green cherries' (those that the informed agents leave for him/her). This is what occurs in the above example (in which $e_{3}^{g}=24$ ). The equilibrium price of both kinds of 'cherries' is higher ( 0.9 instead of 0.75 ) because of the increased quality of the mix received by the uninformed agent.

Eventually, if the endowment of 'green cherries' increases enough, the price of 'green cherries' becomes lower than the price of 'red cherries', and the uninformed agent consumes only 'green cherries' and no 'red cherries'. Such bifurcation occurs for an endowment of 'green cherries' equal to 48 , the solution becoming

$\left\{\begin{array}{l}p^{*}=(1,0.5,0.5) \\ x_{1}^{*}=(4,0,16) \\ x_{2}^{*}=(2,0,8) \\ x_{3}^{*}=(6,12,24) .\end{array}\right.$

Fig. 1 plots the equilibrium prices as a function of agent 3's endowment of 'green cherries'.

If the endowment of 'green cherries' is relatively small, 'green cherries' are traded at a higher price than 'red cherries', and all 


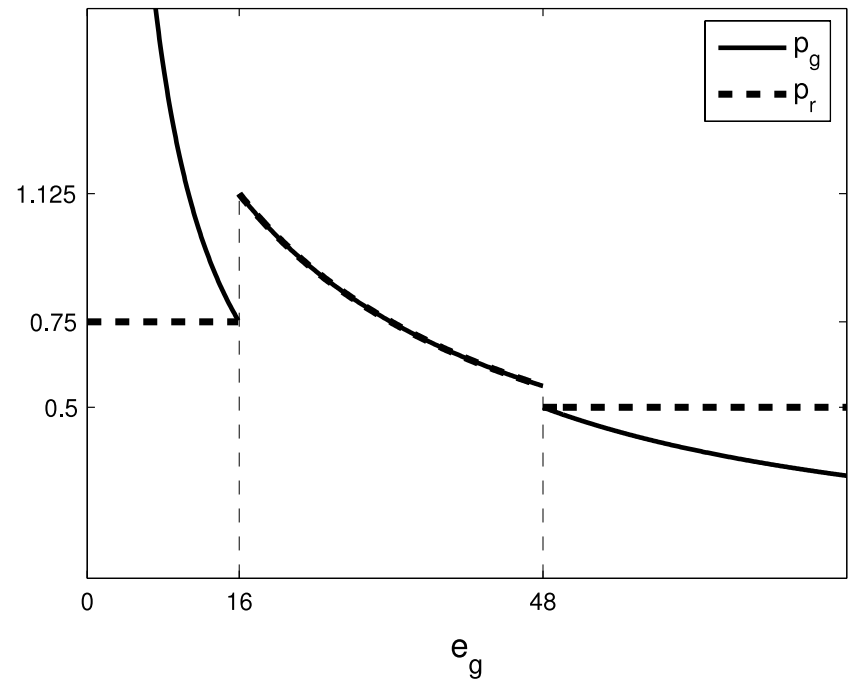

Fig. 1. "Pooling” and "separating" prices.

the supply is absorbed by the informed agents - the uninformed agent is not able to consume any 'green cherries'. If the endowment of 'green cherries' is very high, the opposite occurs. 'Red cherries' are traded at a higher price than 'green cherries', with agent 3 absorbing all the supply, and agent 1 not being able to consume any 'red cherries'. For intermediate values of the supply of 'green cherries', both kinds are traded at the same price, with the informed agents choosing their favorite combination of 'green' and 'red cherries', while the uninformed agent consumes whatever is left.

Regarding the evolution of individual welfare as the endowment of agent 3 increases (Fig. 2), we find that (i) the utility of agent 1 increases until $e_{3}^{g}=37.3$ and then decreases, because after this point the decrease in the price of cherries is not sufficient to compensate for the decrease in the delivery rate of 'red cherries' (which are increasingly scarce); (ii) the utility of agent 2 increases with $e_{3}^{g}$ until $e_{3}^{g}=16$ (because 'green cherries' are becoming cheaper), then it decreases until $e_{3}^{g}=48$ (his/her purchasing power is decreasing as the 'red cherries' are also becoming cheaper), and then it increases again ('green cherries' keep becoming cheaper but now the price of 'red cherries' is remaining constant); (iii) the utility of agent 3 is always increasing with his/her endowment, except at $e_{3}^{g}=48$ (there is a price drop at this point due to the reduction in the demand of agent 1 , who becomes less willing to buy 'cherries' because no 'red cherries' are delivered to him/her).

\section{Welfare}

One of the robust conclusions of the literature on trade under asymmetric information is that information asymmetries entail a welfare cost. This model is not an exception. We find that the equilibrium allocation is not Pareto-optimal and, moreover, that it may be possible to design a system of taxes and subsidies that induces a Pareto-superior allocation using only the information that is common to all the agents.

It is straightforward to show that the equilibrium allocation in the "cherry picking" example is not Pareto-optimal. An improvement would result from the following reallocation: agents 2 and 3 transfer 1 unit of 'cherries' (of any kind, at their discretion) to agent 1 in exchange for 2 units of 'money':

$\left\{\begin{array}{l}x_{1}^{*}=(6,8,0) ; U_{1}^{*}=-\infty \\ x_{2}^{*}=(3,0,6) ; U_{2}^{*}=4.6821 \\ x_{3}^{*}=(3,4,6) ; U_{3}^{*}=6.0684\end{array}\right.$

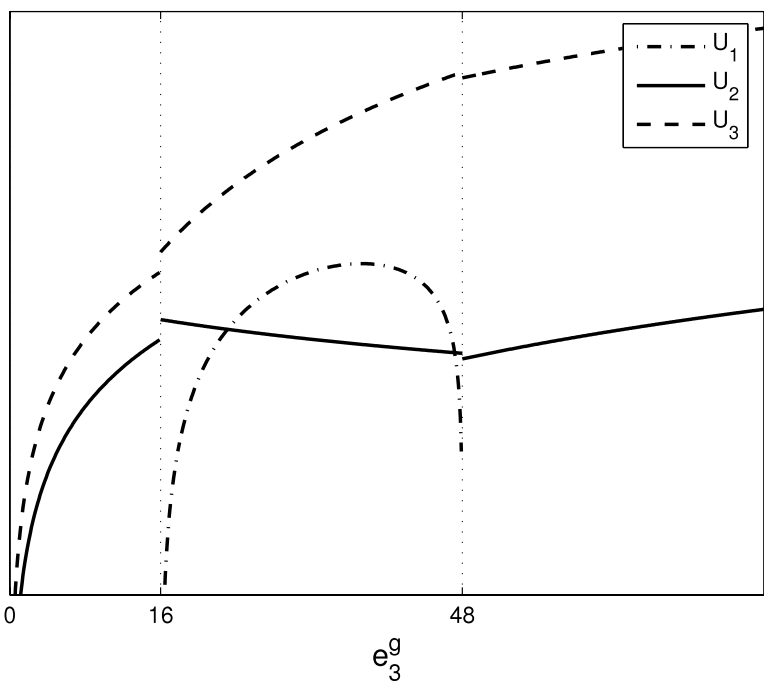

Fig. 2. Individual welfare as a function of $e_{3}^{g}$.

$$
\underset{\text { (reallocation) }}{\Longrightarrow}\left\{\begin{array}{l}
x_{1}^{\prime}=(2,9,1) ; U_{1}^{\prime}=2.8904 \\
x_{2}^{\prime}=(5,0,5) ; U_{2}^{\prime}=4.8283 \\
x_{3}^{\prime}=(5,3,6) ; U_{3}^{\prime}=6.2916 .
\end{array}\right.
$$

A system of anonymous taxes and subsidies can also improve welfare in the "cherry picking" example. Suppose that the government is able to monitor consumption of 'money' and 'cherries' and sets an ad valorem tax, $t$, on the consumption of 'cherries' while subsidizing the consumption of 'money' through an ad valorem subsidy, $s$.

Considering $e_{3}^{g}=15$, without taxes and subsidies the equilibrium allocation is

$\left\{\begin{array}{l}x_{1}^{*}=(6,8,0) ; U_{1}^{*}=-\infty \\ x_{2}^{*}=(3,0,7.5) ; U_{2}^{*}=5.1284 \\ x_{3}^{*}=(3,4,7.5) ; U_{3}^{*}=6.5147 .\end{array}\right.$

Setting $t=0.05$ and $s=0.1113$ (budget-balanced), we obtain (see Appendix A.2)

$\left\{\begin{array}{l}x_{1}^{t}=(3.3756,8.4286,0.2381) ; U_{1}^{t}=0.4780 \\ x_{2}^{t}=(4.4513,0,7.6190) ; U_{2}^{t}=5.5545 \\ x_{3}^{t}=(4.1731,3.5714,7.1429) ; U_{3}^{t}=6.6339 .\end{array}\right.$

A tax on 'cherries' allows agent 1 to consume some 'green cherries' and thus become better off. Agents 2 and 3 also become better off because agent 1 will then be willing to pay more for the 'cherries' (that they are selling).

\section{Concluding remarks}

In an economy in which agents trade goods with uncertain quality, the ability to observe the quality of the good is very useful. To study markets such as the used car market (Akerlof, 1970), it is natural to assume that agents know the quality of the goods that they bring to the market, but have incomplete and differential information about the quality of the goods brought by the other agents.

To model this kind of information asymmetry, we have considered a generalized notion of a good, incorporating in its description the agent that is endowed with this good. This allowed us to study economies in which agents may not have the ability to distinguish good cars from bad cars, but are able to observe the quality of their own cars, or of the cars of their friends. 
Equilibrium is shown to exist, and is characterized by the fact that agents always receive the cheapest delivery that is consistent with their observation abilities (that is, that they cannot distinguish from truthful delivery). We have also found that the law of one price is verified (if two agents sell goods that are undistinguished by the others, then they sell them at the same price). ${ }^{20}$

Finally, it is shown that a budget-balanced system of anonymous taxes and subsidies that only uses information that all agents possess may lead to an allocation that superior in the sense of Pareto.

\section{Appendix}

\section{A.1. Cherry picking with perturbed utility functions}

An objectionable feature of the "cherry picking" example is the fact that the utility functions are not defined in the boundary of the consumption set. Below, we modify the utility functions to overcome this problem, by introducing a parameter, $\epsilon>0$ :

$\left\{\begin{array}{l}U_{1}\left(x_{1}\right)=\ln \left(x_{1}^{0}+\epsilon\right)+\ln \left(x_{1}^{r}+\epsilon\right)+2 \ln \left(x_{1}^{g}+\epsilon\right) \\ U_{2}\left(x_{2}\right)=\ln \left(x_{2}^{0}+\epsilon\right)+2 \ln \left(x_{2}^{g}+\epsilon\right) \\ U_{3}\left(x_{3}\right)=\ln \left(x_{3}^{0}+\epsilon\right)+\ln \left(x_{3}^{r}+\epsilon\right)+2 \ln \left(x_{3}^{g}+\epsilon\right) .\end{array}\right.$

Following the same steps as in the example with $\epsilon=0$, the following demand functions are obtained:

$\left\{\begin{array}{l}x_{2}^{0}=4 p^{r}+\epsilon\left(\frac{p^{g}}{3}-\frac{2}{3}\right) \\ x_{2}^{r}=0 \\ x_{2}^{g}=8 \frac{p^{r}}{p^{g}}+\epsilon\left(\frac{2}{3 p^{g}}-\frac{1}{3}\right) \\ x_{3}^{0}=\frac{e_{3}^{g} p^{g}}{4}+\epsilon\left(\frac{p^{r}}{4}+\frac{p^{g}}{4}-\frac{3}{4}\right) \\ x_{3}^{r}=\frac{e_{3}^{g} p^{g}}{4 p^{r}}+\epsilon\left(\frac{p^{g}}{4 p^{r}}+\frac{1}{4 p^{r}}-\frac{3}{4}\right) \\ x_{3}^{g}=\frac{e_{3}^{g}}{2}+\epsilon\left(\frac{p^{r}}{2 p^{g}}+\frac{1}{2 p^{g}}-\frac{1}{2}\right) .\end{array}\right.$

Suppose that $e_{3}^{g}>48$. In this case (for sufficiently small values of $\epsilon$ ), we must have $p^{g}<p^{r}$ in equilibrium, otherwise the demand of agent 3 for 'red cherries' would be greater than the aggregate supply. This implies that agent 1 is only able to consume 'green cherries'.

Being aware of the fact that he/she will only receive 'green cherries', agent 1 has the following demand:

$\left(x_{1}^{0}, x_{1}^{r}, x_{1}^{g}\right)=\left(4+\epsilon\left(\frac{p^{g}}{3}-\frac{2}{3}\right), 0, \frac{8}{p^{g}}+\epsilon\left(\frac{2}{3 p^{g}}-\frac{1}{3}\right)\right)$.

In equilibrium, demand must equal supply:

$$
\begin{aligned}
& \left\{\begin{array}{l}
x_{1}^{0}+x_{2}^{0}+x_{3}^{0}=12 \\
x_{3}^{r}=12 \\
x_{1}^{g}+x_{2}^{g}+x_{3}^{g}=e_{3}^{g}
\end{array}\right. \\
& \Leftrightarrow\left\{\begin{array}{l}
4+4 p^{r}+\frac{e_{3}^{g} p^{g}}{4}+\epsilon\left(\frac{p^{r}}{4}+\frac{11 p^{g}}{12}-\frac{25}{12}\right)=12 \\
\frac{e_{3}^{g} p^{g}}{4 p^{r}}+\epsilon\left(\frac{p^{g}}{4 p^{r}}+\frac{1}{4 p^{r}}-\frac{3}{4}\right)=12 \\
\frac{8}{p^{g}}+8 \frac{p^{r}}{p^{g}}+\frac{e_{3}^{g}}{2}+\epsilon\left(\frac{11}{6 p^{g}}-\frac{7}{6}+\frac{p^{r}}{2 p^{g}}\right)=e_{3}^{g} .
\end{array}\right.
\end{aligned}
$$

20 The solution concept rules out price discrimination (the price of a good cannot depend on the buyer).
The equilibrium prices are

$\left\{\begin{array}{l}p^{r}=\frac{24 e_{3}^{g}+\epsilon\left(24+7 e_{3}^{g}+9 \epsilon\right)}{48 e_{3}^{g}+\epsilon\left(144+3 e_{3}^{g}+9 \epsilon\right)} \\ p^{g}=\frac{24+6 \epsilon}{e_{3}^{g}+3 \epsilon}\end{array} \Rightarrow\left\{\begin{array}{l}\lim _{\epsilon \rightarrow 0} p^{r}=\frac{1}{2} \\ \lim _{\epsilon \rightarrow 0} p^{g}=\frac{24}{e_{3}^{g}} .\end{array}\right.\right.$

The opposite occurs if we suppose that $e_{3}^{g}<16$. For sufficiently small values of $\epsilon$, we must have $p^{r}<p^{g}$ in equilibrium, otherwise $x_{2}^{g}+x_{3}^{g}>e_{3}^{g}$. In this case, agent 1 is only able to consume 'red cherries'.

The resulting demand of agent 1 is

$$
\left(x_{1}^{0}, x_{1}^{r}, x_{1}^{g}\right)=\left(6+\epsilon\left(\frac{p^{r}}{2}-\frac{1}{2}\right), \frac{6}{p^{r}}+\epsilon\left(\frac{1}{2 p^{r}}-\frac{1}{2}\right), 0\right) .
$$

And the equilibrium prices are

$$
\left\{\begin{array} { l } 
{ p ^ { r } = \frac { 3 6 e _ { 3 } ^ { g } + \epsilon ( 6 0 + 8 e _ { 3 } ^ { g } + 1 1 \epsilon ) } { 4 8 e _ { 3 } ^ { g } + \epsilon ( 9 6 + 6 e _ { 3 } ^ { g } + 1 1 \epsilon ) } } \\
{ p ^ { g } = \frac { 5 7 6 + \epsilon ( 2 7 6 + 2 2 \epsilon ) } { 4 8 e _ { 3 } ^ { g } + \epsilon ( 9 6 + 6 e _ { 3 } ^ { g } + 1 1 \epsilon ) } }
\end{array} \Rightarrow \left\{\begin{array}{l}
\lim _{\epsilon \rightarrow 0} p^{r}=\frac{3}{4} \\
\lim _{\epsilon \rightarrow 0} p^{g}=\frac{12}{e_{3}^{g}} .
\end{array}\right.\right.
$$

Finally, suppose that $16<e_{3}^{g}<48$. For sufficiently small values of $\epsilon$, we must have $p^{r}=p^{g}$ in equilibrium. ${ }^{21}$ In this case, utility maximization by agent 1 implies that

$\frac{1}{x_{1}^{0}+\epsilon}=\left[\frac{1-k^{g}}{\left(1-k^{g}\right) x_{1}^{r g}+\epsilon}+\frac{2 k^{g}}{k^{g} x_{1}^{r g}+\epsilon}\right] \frac{1}{p^{r g}}$,

where $x_{1}^{r g}$ is the quantity of cherries that agent 1 buys, $p^{r g}$ is the price of cherries and $k^{g}$ is the delivery rate of 'green cherries'.

Together with the budget restriction $\left(x_{1}^{0}+x_{1}^{r g} p^{r g}=12\right)$, the above condition allows us to find the demand of agent 1 :

$x_{1}^{0}=\frac{15}{2}+\epsilon\left[\frac{2+k^{g}}{8 k^{g}\left(1-k^{g}\right)} p-\frac{3}{8}\right]$

$-\sqrt{\left\{-\frac{9}{2}+\epsilon\left[\frac{2+k^{g}}{8 k^{g}\left(1-k^{g}\right)} p-\frac{3}{8}\right]\right\}^{2}+\frac{\epsilon p\left[12\left(1+k^{g}\right)+\epsilon\left(1+k^{g}-p\right)\right]}{4 k^{g}\left(1-k^{g}\right)} .}$

Assuming that $k^{g}$ converges to a value in $(0,1)$, we conclude that $\lim _{\epsilon \rightarrow 0} x_{1}^{0}=3$ and, therefore, $\lim _{\epsilon \rightarrow 0} x_{1}^{r g}=\frac{9}{p}$.

Since $\lim _{\epsilon \rightarrow 0}\left(x_{1}^{0}+x_{2}^{0}+x_{3}^{0}\right)=3+4 p^{r g}+\frac{e_{3}^{g} p^{r g}}{4}$, we must have

$\lim _{\epsilon \rightarrow 0}\left(3+4 p^{r g}+\frac{e_{3}^{g} p^{r g}}{4}\right)=12 \Rightarrow \lim _{\epsilon \rightarrow 0} p^{r g}=\frac{36}{16+e_{3}^{g}}$.

The associated limit allocation is

$$
\begin{aligned}
& \left(x_{1}^{0}, x_{1}^{r}, x_{1}^{g}\right)=\left(3,12-\frac{e_{3}^{g}}{4}, \frac{e_{3}^{g}}{2}-8\right) \\
& \left(x_{2}^{0}, x_{2}^{r}, x_{2}^{g}\right)=\left(\frac{144}{16+e_{3}^{g}}, 0,8\right) \\
& \left(x_{3}^{0}, x_{3}^{r}, x_{3}^{g}\right)=\left(\frac{9 e_{3}^{g}}{16+e_{3}^{g}}, \frac{e_{3}^{g}}{4}, \frac{e_{3}^{g}}{2}\right) .
\end{aligned}
$$

21 Otherwise, if $p^{r}<p^{g}$, then $x_{1}^{g}=0$ and there would be excess supply of 'green
cherries'; if $p^{g}<p^{r}$, then $x_{1}^{r}=0$ and there would be excess supply of 'red cherries'. 


\section{A.2. Cherry picking with taxes and subsidies}

Consider an ad valorem tax, $t$, on the consumption of 'cherries' and an ad valorem subsidy, $s$, to the consumption of 'money'.

Combining the first-order condition for utility maximization with the budget restriction, we find the demand of agent 2 :

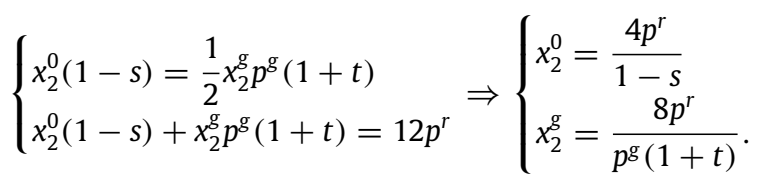

Similarly, we find the demand of agent 3:

$$
\begin{aligned}
& \left\{\begin{array}{l}
x_{3}^{0}(1-s)=x_{3}^{r} p^{r}(1+t) \\
x_{3}^{r} p^{r}=\frac{1}{2} x_{3}^{g} p^{g} \\
x_{3}^{0}(1-s)+x_{3}^{r} p^{r}(1+t)+x_{3}^{g} p^{g}(1+t)=e_{3}^{g} p^{g}
\end{array}\right. \\
& \Rightarrow\left\{\begin{array}{l}
x_{3}^{0}=\frac{e_{3}^{g} p^{g}}{4(1-s)} \\
x_{3}^{r}=\frac{e_{3}^{g} p^{g}}{4 p^{r}(1+t)} \\
x_{3}^{g}=\frac{e_{3}^{g}}{2(1+t)} .
\end{array}\right.
\end{aligned}
$$

An equilibrium condition is that

$x_{2}^{g}+x_{3}^{g} \leq e_{3}^{g} \Leftrightarrow \frac{8 p^{r}}{p^{g}(1+t)}+\frac{e_{3}^{g}}{2(1+t)} \leq e_{3}^{g}$.

Considering $e_{3}^{g}=15$ and $t=0$, the above condition becomes

$\frac{p^{r}}{p^{g}} \leq \frac{15}{16}$

implying that agent 1 does not receive any 'green cherries'.

In that case (no taxes), the equilibrium allocation is

$$
\left\{\begin{array}{l}
x_{1}^{*}=(6,8,0) ; U_{1}^{*}=-\infty \\
x_{2}^{*}=(3,0,7.5) ; U_{2}^{*}=5.1284 \\
x_{3}^{*}=(3,4,7.5) ; U_{3}^{*}=6.5147 .
\end{array}\right.
$$

Maintaining $e_{3}^{g}=15$ but now with $t=0.05$, we find that the demand of agents 2 and 3 for 'green cherries' is no longer sufficient to absorb all the supply at $p^{g}<p^{r}$ :

$p^{g}<p^{r} \Rightarrow x_{2}^{g}+x_{3}^{g}<\frac{8}{1.05}+\frac{15}{2 * 1.05}=14.7619$.

Given that agent 1 receives some 'green cherries' and some 'red cherries':

$$
\begin{aligned}
U_{1}\left(x_{1}^{0}, x_{1}^{r g}\right)= & \ln \left(x_{1}^{0}\right)+\ln \left(\left(1-k^{g}\right) x_{1}^{r g}\right) \\
& +2 \ln \left(k^{g} x_{1}^{r g}\right) \Rightarrow \frac{\partial U_{1}}{\partial x_{1}^{r g}}=\frac{3}{x_{1}^{r g}} .
\end{aligned}
$$

Therefore, the demand of agent 1 is obtained as follows:

$$
\left\{\begin{array} { l } 
{ x _ { 1 } ^ { 0 } ( 1 - s ) = \frac { 1 } { 3 } x _ { 1 } ^ { r g } p ^ { r g } ( 1 + t ) } \\
{ x _ { 1 } ^ { 0 } ( 1 - s ) + x _ { 1 } ^ { r g } p ^ { r g } ( 1 + t ) = 1 2 }
\end{array} \Rightarrow \left\{\begin{array}{l}
x_{1}^{0}=\frac{3}{1-s} \\
x_{1}^{r g}=\frac{9}{p^{r g}(1+t)} .
\end{array}\right.\right.
$$

For the budget to be balanced, it is necessary that $s=\frac{12 p^{r}+e_{3}^{g} p^{g}}{12} t$. In this case $s=\frac{27 p^{r g}}{12} t$.

The equilibrium prices can be obtained by equating the demand and the supply of 'cherries':

$$
\begin{aligned}
x_{1}^{r g}+x_{2}^{r g}+x_{3}^{r g}= & 27 \Leftrightarrow \frac{9}{p^{r g}(1+t)}+\frac{8}{1+t}+\frac{15}{4(1+t)} \\
& +\frac{15}{2(1+t)}=27 \Leftrightarrow p^{r g}=\frac{36}{31+108 t} .
\end{aligned}
$$

With $t=0.05$ and $s=0.1113$, we obtain $p^{r g}=0.9890$ and

$$
\left\{\begin{array}{l}
x_{1}^{t}=(3.3756,8.4286,0.2381) ; U_{1}^{t}=0.4780 \\
x_{2}^{t}=(4.4513,0,7.6190) ; U_{2}^{t}=5.5545 \\
x_{3}^{t}=(4.1731,3.5714,7.1429) ; U_{3}^{t}=6.6339
\end{array}\right.
$$

\section{References}

Aliprantis, C.D., Border, K.C., 2006. Infinite Dimensional Analysis, third ed.. SpringerVerlag, Berlin.

Akerlof, G.A., 1970. Market for lemons: quality uncertainty and the market mechanism. Quarterly Journal of Economics 84 (3), 488-500.

Bisin, A., Gottardi, P., 1999. Competitive equilibria with asymmetric information. Journal of Economic Theory 87 (1), 1-48.

Bisin, A., Gottardi, P., 2006. Efficient competitive equilibria with adverse selection Journal of Political Economy 114 (3), 485-516.

Correia-da-Silva, J., Hervés-Beloso, C., 2008. Subjective expectations equilibrium in economies with uncertain delivery. Journal of Mathematical Economics 44 (7-8), 641-650.

Correia-da-Silva, J., Hervés-Beloso, C., 2009. Prudent expectations equilibrium in economies with uncertain delivery. Economic Theory 39 (1), 67-92.

Correia-da-Silva, J., Hervés-Beloso, C., 2011, General equilibrium with uncertain delivery, Economic Theory, forthcoming (http://dx.doi.org/10.1007/s00199011-0647-1).

Dubey, P., Geanakoplos, J., 2002. Competitive pooling: Rothschild-Stiglitz reconsidered. Quarterly Journal of Economics 117 (4), 1529-1570.

Dubey, P., Geanakoplos, J., Shubik, M., 2005. Default and punishment in general equilibrium. Econometrica 73 (1), 1-37.

Gale, D., 1992. A walrasian theory of markets with adverse selection. Review of Economic Studies 59, 229-255.

Gale, D., 1996. Equilibria and pareto optima of markets with adverse selection. Economic Theory 7, 207-235.

Meier, M., Minelli, E., Polemarchakis, H.M., 2011. Competitive Markets with Private Information on Both Sides. mimeo.

Minelli, E., Polemarchakis, H.M., 2000. Nash-Walras equilibria of a large economy. Proceedings of the National Academy of Sciences of the United States of America 97 (10), 5675-5678.

Prescott, E.C., Townsend, R.M., 1984a. Pareto optima and competitive equilibria with adverse selection and moral hazard. Econometrica 52 (1), 21-46.

Prescott, E.C., Townsend, R.M., 1984b. General competitive analysis in an economy with private information. International Economic Review 25 (1), 1-20.

Rustichini, A., Siconolfi, P., 2008. General equilibrium in economies with adverse selection. Economic Theory 37 (1), 1-29. 